\title{
Time-saving method of orbital thermal regime calculations of nanosatellites as exemplified by a 3U CubeSat
}

\author{
Vasily Gorev ${ }^{1, *}$, Vitaly Prokopyev ${ }^{1}$, Yury Prokopyev $^{1}$, and Alexey Sidorchuk ${ }^{1,2}$ \\ ${ }^{1}$ Novosibirsk State University, 630090, Pirogova str., 2, Novosibirsk, Russia \\ ${ }^{2}$ OKB Fifth Generation Ltd., 630090, Nikolaeva str., 11, Novosibirsk, Russia
}

\begin{abstract}
A time-saving approach to perform technical calculations of thermal conditions of orbital motion of $3 \mathrm{U}$ CubeSat nanosatellite was applied, which made it possible to make the thermal calculations of a satellite with simple structure geometry using MatLab and SolidWorks Simulation. Passive thermal regulation facilities are sufficient for a $3 \mathrm{U}$ CubeSat to provide thermal conductivity of the case's structural elements and to remove heat from the lighted surface and internal components to the satellite's shadowed surface. Application of spectrally selective coatings allows narrowing the range of surface temperatures of $3 \mathrm{U}$ CubeSat.
\end{abstract}

\section{Introduction}

The design of the thermal operating conditions is one of the main tasks in satellite development procedure. For the regular operation of the satellite equipment, it is necessary to maintain the thermal conditions of the satellite functional elements in a certain narrow operational range against the extreme conditions realized in space. The initial requirements for the design of the satellite's thermal regime are the conditions of its operation in space, its power-to-weight ratio, and the operating modes of the heat producing parts [1].

All space equipment requires a precise development because of impossibility of any kind of reparations after launch. Therefore, comprehensive ground-based tests of onboard systems as well as structural elements of satellites, computational techniques come into importance. They make it possible to optimize many parameters of the device on early stages of design. Calculating the thermal conditions of the spacecraft is a standard and required task; there is a range of instruments for performing such calculations. There are also specialized software packages for calculations of radiative heat transfer, such as Thermal Desktop, ESARAD / ESATAN TMS [2] and other software products. In general, in the family of software products for scientific and technical calculations, there is a tendency splice it with MCAD development environments, thus creating a unified MCAD development environment with numerical studies facilities. This fact is caused by desire to automate the mostly manual transfer of product geometry from a development software to a calculation program.

\footnotetext{
* Corresponding author: vasily.gorev@gmail.com
} 
This paper discusses the problem of developing a time-saving method for in-orbit thermal conditions calculation of nanosatellite. It is proposed to stay within capabilities of MCAD environment where the spacecraft is being developed, i.e., SolidWorks Simulation. This approach makes it possible to reduce significantly the time for transferring and adapting the satellite model geometry to calculation program. Nevertheless, it is still sufficient for structure development to obtain a necessary information about thermal conditions of nanosatellite.

\section{Calculation procedure}

\subsection{Calculation of radiation absorbed by a satellite during orbital motion}

The procedure for calculating the nanosatellite thermal conditions consists of two parts:

1) calculating the power absorbed by the faces of a satellite performed in the MatLab environment;

2) calculating the heat transfer under structural elements of the case of a satellite and the heat dissipation in the outer space realized using the SolidWorks MCAD tool - SolidWorks Simulation.

During the first step of the procedure, when modeling the satellite radiation conditions, a circular Low Earth Orbit was considered; the main external parameters of the task are the altitude $H$ of the orbit and the angle $B$ between the satellite's orbit plane and the direction to the Sun $[1,3]$. For this calculation, $H$ is assumed $450 \mathrm{~km}$ that approximately corresponds to the International Space Station orbit.

Let us consider the orientation where the satellite will be directed by a small face of the $1 \mathrm{U}$ case toward the Earth, and one of the large lateral $3 \mathrm{U}$ faces will be perpendicular to the orbital velocity (Figure 1, a). To calculate a satellite insolation regime, all elements protruding from the case, such as antennas and folding panels of solar batteries, are ignored. The intensity of absorbed power is calculated on the satellite case outer faces for various materials: anodized aluminum alloy, photovoltaic converters, and the face panels' surface covered with the spectral-selective paint.
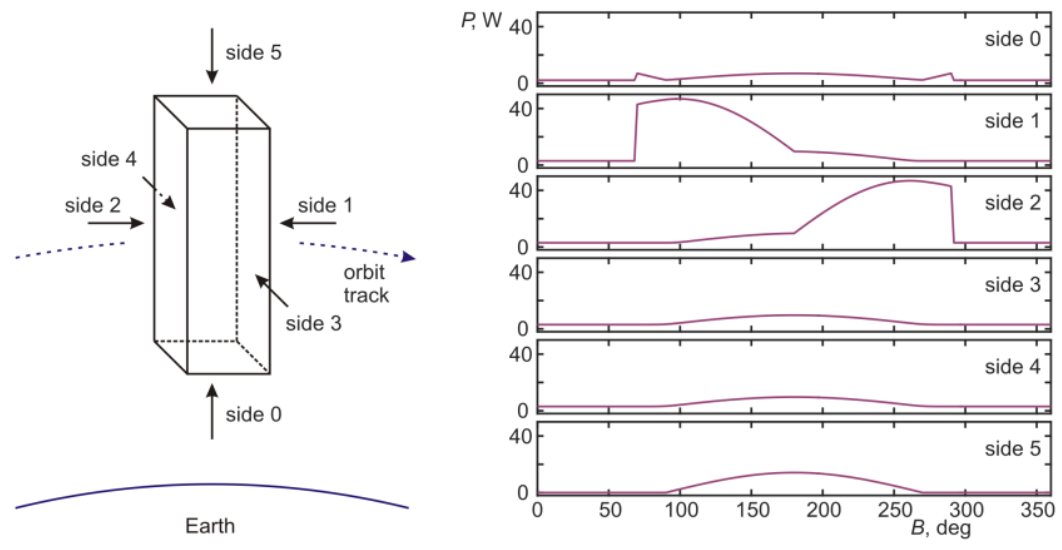

Fig. 1. Earth-pointing and relative motion (left) orientation of the $3 \mathrm{U}$ structure. Total incident power radiation (right) applied to satellite surfaces, depending on the position in orbit at $B=0^{\circ}$.

The calculation takes into account three radiation sources: the solar radiation, the Earth's albedo, and the Earth IR radiation. The solar radiation is calculated as the radiation of a black body with a temperature of $5762 \mathrm{~K}$, which approximates the solar radiation spectrum 
with the sufficiently high accuracy [4]. The solar radiation average intensity is assumed to be $1367 \mathrm{~W} / \mathrm{m}^{2}$ [1]. To calculate the magnitude of the radiation reflected by the Earth, the approximate calculation method recommended in work [4] was applied. This method conforms the experimental data obtained within the framework of the Earth Radiation Budget Experiment (ERBE) project $[1,5]$. The value of the Earth infrared radiation is assumed to be $245 \mathrm{~W} / \mathrm{m}^{2}$ at the altitude of $30 \mathrm{~km}$ above the Earth's surface $[1,5]$, and it is recalculated for the device orbit altitude.

\subsection{CubeSat calculation models}

To calculate the thermodynamics of the nanosatellite, several CAD models of the CubeSat with various simplifications regarding the actual design of the $3 \mathrm{U}$ satellite available at this design stage was developed. Thermal conditions were calculated for models of different design simplification degree (from simple to complex).

The first simplified 3U-type model (model-1) is a single body combining frames and face panels made of anodized 2024 aluminum alloy; the absorption coefficients of solar radiation and the IR emission are taken from [6, 7]. On the outer surface of the model, photovoltaic modules are located, as the isolated parts of the surface. Inside the model, there are no electronic components and boards, only structural elements for their fastening (Figure 2, a). This approach simulates the design of the spacecraft in which aluminum panels coated with a thin layer of the copper-plated FR-4 are used - a common material for PCB, with high heat dissipation (HA80 and other similar materials), applied, for example, in LED lamps. In terms of the thermal conditions of the spacecraft and photovoltaic modules, using the FR-4 or aluminum-based panels can have a qualitatively different effect. In the case of aluminum panels, a high thermally conductive case minimizes the instantaneous case temperature difference; it conducts the heat from the illuminated sides to the unlit ones, and thus it facilitates the effective heat dissipation in space.
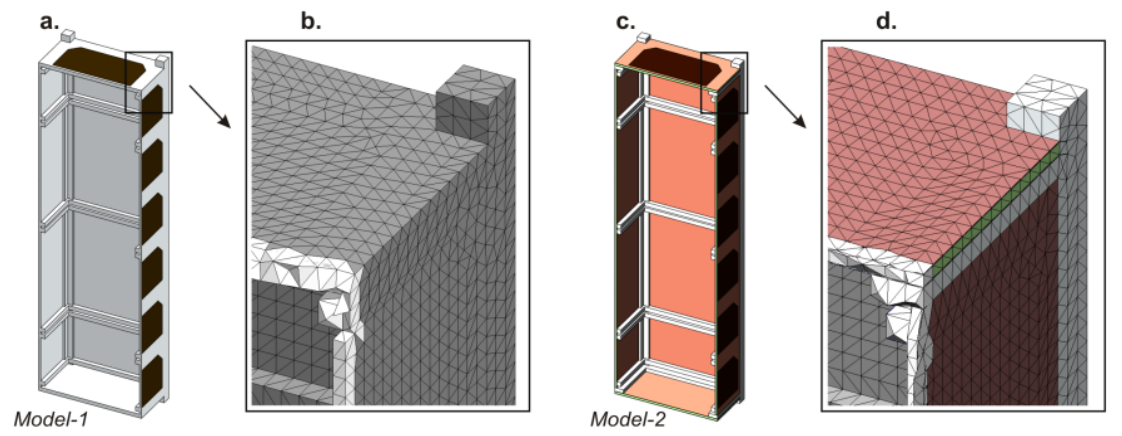

Fig. 2. CubeSat $3 U$ case models. A one-piece case made of aluminum alloy (a) and its finite element mesh (b), a case made of copper-plated FR-4 panels on an aluminum alloy frame (c) and its finite element mesh (d).

On the other hand, the FR-4 can be much more convenient when installing the electronic components on the solar panel to control the inclusion of batteries in the satellite power supply system. Further, on model-2 with the side panels made of copper-plated FR-4 (Figure 2, c) was considered. The panel consists of three layers: a fiberglass composite layer $1.6 \mathrm{~mm}$ thick is coated on both sides with layers of $0.2 \mathrm{~mm}$ copper. In the real construction, the FR-4 can have two, four or more layers of copper of smaller thickness. The panels' thermodynamics at the typical time of this heat exchange process is twodimensional with good accuracy, so only the total thickness of the copper layer and the fiberglass composite layer is important. The calculated grid on thin planar elements has one 
cell in thickness direction, i.e., it is two-dimensional (Figure 2, d). The thermal resistance of the contacts between the details is not taken into account while calculating.

\section{Results and discussions}

The CubeSat dynamic calculation of the thermal conditions of model-1 was made for the orbits corresponding to the values of the angle $B=0^{\circ}, 45^{\circ}, 70^{\circ}$ (Figure 3 ). The $B$ angle $=$ $70^{\circ}$ corresponds to the point of the maximum average-orbital temperature for the $H$ orbit altitude $=450 \mathrm{~km}$ for the studied satellite orientation in space, because it is the smallest one among the $B$ angles, at which the satellite does not enter the Earth shadow. As the initial conditions for dynamic calculations, one uses the solution of the stationary problem for the equilibrium case at the initial point of the path - in the middle of the orbital eclipse period.

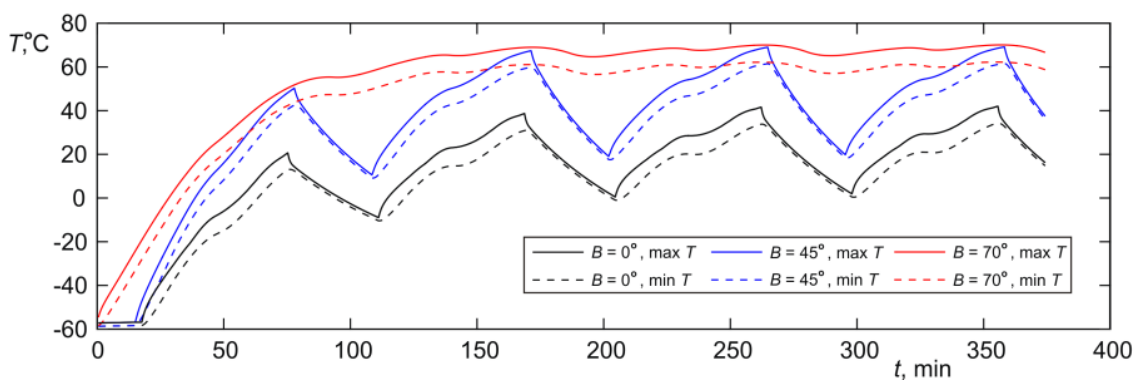

Fig. 3. Graphs of the minimum and maximum satellite surface temperatures for orbits with $B=0^{\circ}$, $45^{\circ}, 70^{\circ}$.

Figure 3 shows the graphs of the minimum and maximum temperatures of the outer surface of the satellite model's case. The initial part of the graph corresponds to the transient process from the equilibrium case for the orbital eclipse period of the initial conditions to the satellite's periodic thermal conditions. In the steady-state periodic part of the graph one can see the features of the thermal conditions associated with the shape and orientation of the satellite (they are clearly seen in the graphs for $B=0^{\circ}$ ); in the areas of the spacecraft temperature growth, observing a bend corresponding to a decrease in insolation associated with the orientation of the satellite by a small face on the Sun. The temperature fluctuations in the case of the $B$ angle $B 70^{\circ}$ are not big, because the spacecraft on this orbit doesn't enter the shadow of the Earth, and they are caused by a change in the orientation of the spacecraft relative to the Sun and by the periodic illumination from the Earth's albedo. One should note that the difference between the minimum and maximum instantaneous temperature of model- 1 does not exceed $10^{\circ} \mathrm{C}$, which is achieved due to the high thermal conductivity of the satellite's case.

For model-2 of the spacecraft, two versions of the thermal calculations for the steadystate orbital case concerning the satellite thermodynamics for $B=90^{\circ}$ was made (Figure 4): 1). The side panels are made of $2 \mathrm{~mm}$ fiberglass composite; there is no copper deposition (Figure 4, b). In this case, the heat removal from the illuminated panel to the rest panels is difficult due to the low thermal conductivity of the fiberglass composite, observing some areas with a temperature exceeding $200^{\circ} \mathrm{C}$ on the illuminated surface. The temperature maxima are located between the aluminum fins of the frame, to which the panel adjoins. The absorption coefficient of the sunlight range and the emission factor of the IR radiation of the panel surfaces are assumed to be equal to the parameters of the 2024 anodized aluminum alloy (shown in Fig. 4. 2). The side panels are made of copper-plated FR-4, as described above. Two copper layers with a total thickness of $0.4 \mathrm{~mm}$ on the side panel reduce the maximum temperature of the illuminated side of the satellite to $60^{\circ} \mathrm{C}$ (Figure 4 , 
c), which is close to the case of model-1 for the same orbit (Figure 4, a). Figure 4, d shows the surface temperature field of the CubeSat model for the case identical to the one shown in Figure 4, c, except for the optical properties of the surface of the side panels.

Assuming the coefficient of the sunlight absorption and the IR radiation emission factor to be equal to the parameters of the spectral-selective paint (Figure 4, d). Covering the panels with the selective paint in the intervals between photovoltaic modules makes it is possible to decrease the upper limit of the temperature range of the satellite's case up to $25^{\circ} \mathrm{C}$, the lower one to $10^{\circ} \mathrm{C}$.
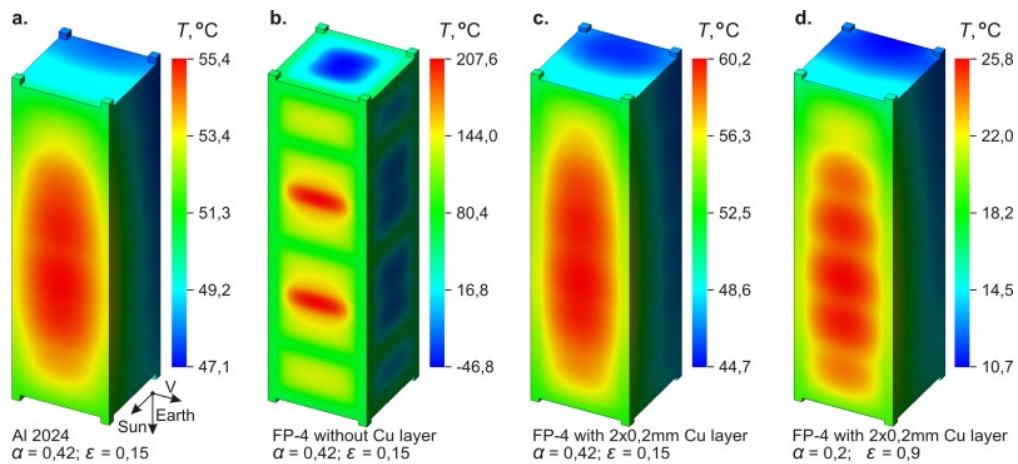

Fig. 4. Spacecraft model surface temperature fields. A solid case made of aluminum alloy (a), a case made of FR-4 panels without copper-plating (b), with copper-plating on an aluminum alloy frame (c). Case (c), covered with spectral-selective paint (d).

\section{Conclusion}

The time-saving method of technical calculations of thermal conditions is described as exemplified by $3 \mathrm{U}$ CubeSat nanosatellite with simplified structure geometry. It consists of two procedures: calculating the boundary conditions for the surface elements in the MatLab software package and calculating the thermal conductivity of the satellite structure and the IR radiation of the satellite's surface via SolidWorks Simulation.

The characteristic time of radiation thermal stabilization for $3 \mathrm{U}$ CubeSat is comparable with the period at Low Earth Orbit. The time of satellite surface temperature equilibrium setting is much less then orbital period for the case of $2 \mathrm{~mm}$ thick aluminum alloy face panels or FR-4 face panels with $0.4 \mathrm{~mm}$ total thickness of copper layer. Therefore, passive facilities are sufficient for $3 \mathrm{U}$ CubeSat thermoregulation.

Application of spectrally selective coatings allows narrowing the range of surface temperatures of $3 \mathrm{U}$ CubeSat. Coating with $\alpha=0.2, \varepsilon=0.9$, instead of the anodized 2024 alloy $(\alpha=0.43, \varepsilon=0.15)$, reduces the maximum surface temperature of the satellite from $60.2^{\circ} \mathrm{C}$ to $25.8^{\circ} \mathrm{C}$.

This work was supported by the Ministry of Education and Science of the Russian Federation: Project No. 14.575.21.0154, Project Identification No. RFMEFI57517X0154.

\section{References}

1. Spacecraft thermal control handbook. Volume I: Fundamental technologies (Edited by D. G. Gilmore, The Aerospace Press, 2002)

2. K. Yang, TFAWS, Pasadena, CA, (2012) 
3. Space Mission Engineering: The New SMAD (Edited by J. R. Wertz, Microcosm Press, 2011)

4. R. E. Smith, G. S. West, NASA Technical Memorandum 82478 (1983)

5. B. R. Barkstrom, E. F. Harrison, G. L. Smith, R. D. Cess, JASR 9, 7, 75 (1989)

6. J. H. Henninger, NASA Reference Publication 1121 (1984)

7. J. L. Golden, NASA Conference Publication 3257, 61 (1992) 